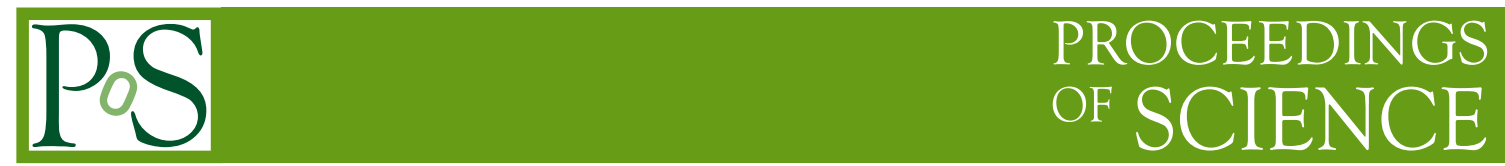

\title{
Lorentz boosts in interacting systems
}

\author{
Eugene V. Stefanovich* \\ Mountain View, USA \\ E-mail: eugene stefanovichdusa.net
}

In quantum systems interaction is normally modeled by adding a potential energy operator to the generator of time translations - the Hamiltonian. It is less known that in order to maintain the Poincare invariance, a special interaction operator must be also added to the generator of Lorentz boosts. In this article we will discuss possible observable consequences of such "boost interactions".

XXII International Baldin Seminar on High Energy Physics Problems,

15-20 September 2014

JINR, Dubna, Russia

${ }^{*}$ Speaker. 


\section{Relativistic Hamiltonian theories}

Since seminal work of Eugene Wigner [四, we know that any relativistic quantum theory must have an unitary representation $U_{g}$ of the Poincaré group in its Hilbert space. Elementary particles are identified with irreducible unitary representations of the Poincaré group. Hilbert spaces of compound multiparticle systems are constructed as (properly symmetrized and/or antisymmetrized) tensor products of one-particle spaces. Ten Hermitian generators of the representation $U_{g}$ are interpreted as total observables of the system: the total momentum $\mathbf{P}$, the total energy (Hamiltonian) $H$, etc. A special role is played by the boost operator $\mathbf{K}$, which controls transitions between reference frames moving with different velocities. Usually, the latter transformations are relegated to formulas from Einstein's special relativity. In Wigner's theory we have a chance to verify these formulas.

\subsection{Boost transformations in non-interacting systems}

The simplest example of a relativistic quantum theory is a system of two non-interacting massive spinless particles in one spatial dimension. Three Poincaré generators are defined as sums of one-particle observables

$$
\begin{aligned}
P_{0} & =p_{1}+p_{2} \\
H_{0} & =h_{1}+h_{2} \\
K_{0} & =k_{1}+k_{2}
\end{aligned}
$$

while particle positions are represented by Newton-Wigner operators [[]]

$$
x_{i}=-\frac{c^{2}}{2}\left(h_{i}^{-1} k_{i}+k_{i} h_{i}^{-1}\right), \quad i=1,2
$$

In the rest frame $O$ one can apply time translation to these observables and obtain their usual linear time dependencies

$$
x_{i}(t)=e^{\frac{i}{\hbar} H_{0} t} x_{i} e^{-\frac{i}{\hbar} H_{0} t}=x_{i}+\frac{p_{i} c^{2}}{h_{i}} t=x_{i}+v_{i} t
$$

where $v_{i}=p_{i} c^{2} / h_{i}$ are the velocity operators of the two particles. For further analysis it is convenient to take the quasiclassical limit in which particle states are represented by localized wave packets that follow classical trajectories. Then formulas (ㅁ. $\mathbb{D}$ ) confirm that non-interacting classical particles move along straight lines with constant velocities $v_{i}$. The point of intersection of the two trajectories can be found as the solution of equation $x_{1}(t)=x_{2}(t) \equiv x$. This point marks a localized event $E$ with well-defined time and position $(x, t)$. See Fig. 凹 (a).

Using the same approach, we can apply a boost transformation

$$
x_{i}(\theta, t)=e^{-\frac{i}{\hbar} K_{0} c \theta} x_{i}(t) e^{\frac{i}{\hbar} K_{0} c \theta}
$$




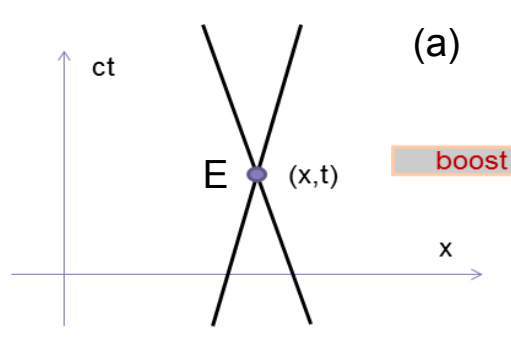

(a)

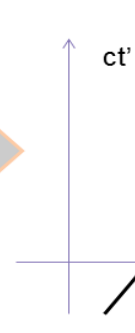

(b)

$\left(x^{\prime}, t^{\prime}\right)$
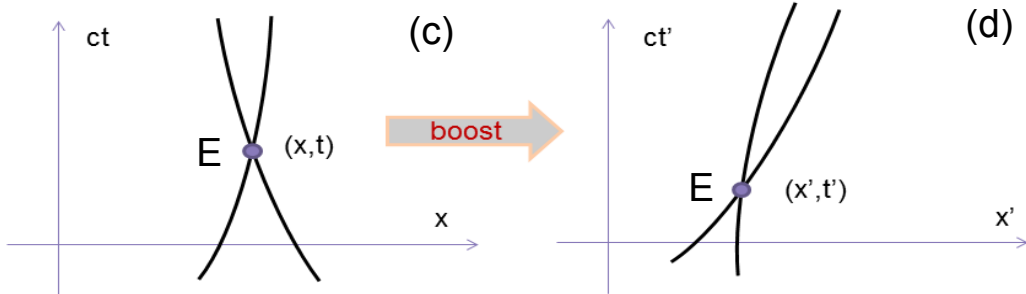

(d)

Figure 1: Boost transformations of particles' trajectories: (a) - (b) non-interacting case; (c) - (d) interacting case.

to find out how particle trajectories look from a reference frame $O^{\prime}$ moving with velocity $c \tanh \theta$. Then, solving equation $x_{1}^{\prime}\left(\theta, t^{\prime}\right)=x_{2}^{\prime}\left(\theta, t^{\prime}\right) \equiv x^{\prime}$ we obtain space-time coordinates $\left(x^{\prime}, t^{\prime}\right)$ of the event $E$ in the moving frame. See Fig. $\mathbb{W}$ (b). A simple algebra yields the relationship between the primed and non-primed sets of coordinates. These are the well-known Lorentz transformations for the time and position

$$
\begin{aligned}
x^{\prime} & =x \cosh \theta-c t \sinh \theta \\
t^{\prime} & =-(x / c) \sinh \theta+t \cosh \theta
\end{aligned}
$$

This implies that all formulas of Einstein's special relativity are valid for events associated with non-interacting particles [3]].

\subsection{Boost transformations in interacting systems}

In order to apply a similar analysis to a system of interacting particles we first need to specify the Poincaré group representation describing this system. In the instant form of Dirac's dynamics [四], generators of both time translations and boosts become modified by the addition of interaction operators

$$
\begin{aligned}
P & =P_{0} \\
H & =H_{0}+V \\
K & =K_{0}+Z
\end{aligned}
$$


Note that one cannot add interaction $V$ only to the Hamiltonian, as it was done in non-relativistic physics. It is important that the boost interaction $Z$ is present as well, otherwise we cannot guarantee the commutation relations of the Poincare Lie algebra. Although the presence of the boost interaction is well known, its observational consequences have not been properly analyzed yet.

Just as in $(\mathbb{L})$, we obtain rest-frame trajectories by applying time translations with the interacting Hamiltonian

$$
x_{i}(t)=e^{\frac{i}{\hbar} H t} x_{i} e^{-\frac{i}{\hbar} H t}=x_{i}+v_{i} t+\frac{a_{i}}{2} t^{2}+\ldots
$$

As expected, due to the non-trivial interaction between our particles, their trajectories are no longer uniform and linear. The accelerations $a_{i}$ can be written down only if we know the explicit form of the interaction operator $V$ and the initial state of the system. Then one can also find the point of intersection of the two trajectories and thus define a localized event $E$ with its space-time coordi-

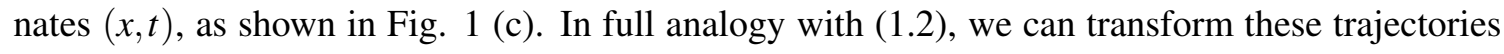
to the moving frame by applying the interacting boost operator

$$
x_{i}(\theta, t)=e^{-\frac{i}{\hbar} K c \theta} x_{i}(t) e^{\frac{i}{\hbar} K c \theta}
$$

and obtain space-time coordinates $\left(x^{\prime}, t^{\prime}\right)$ of the event $E$ in the moving frame. See Fig. W(d). Unlike in the non-interacting case, now there is no simple and unique transformation formula between the primed and non-primed sets of coordinates. The relationship between these two sets depends on the interaction operators $V$ and $Z$ as well as on the states of involved particles.

The famous Currie-Jordan-Sudarshan theorem [5] makes an even stronger statement: there can be no relativistic interacting multi-particle system, whose trajectories transform by Lorentz formulas (L.3) - (L4).

\subsection{Superluminal signals and causality}

Einstein's special relativity claims the impossibility of superluminal propagation of particles and signals. This claim is usually based on the principle of causality, which says that the cause precedes the effect in all reference frames. Suppose that two events "Cause" and "Effect" are causally related, while separated by a space-like interval in the reference frame $O$ with coordinate

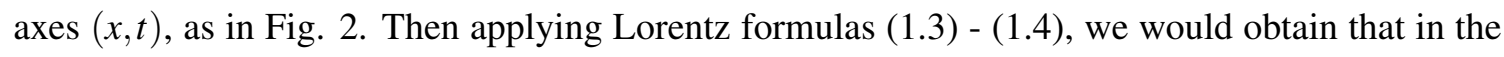
moving reference frame $O^{\prime}$ with axes $\left(x^{\prime}, t^{\prime}\right)$ the "Effect" happened earlier than the "Cause," which would violate the principle of causality.

However, as we already established, Lorentz formulas are no longer valid for events occurring in interacting multiparticle systems. Thus, the "pseudorotation" of the coordinate axes $(x, t) \rightarrow$ $\left(x^{\prime}, t^{\prime}\right)$ in Fig. $\square$ is not an accurate representation of the boost transformation, and the ban on superluminal signals cannot be proven rigorously.

\section{Examples of interacting relativistic systems}

In the preceding section we advanced some theoretical arguments suggesting that formulas of Einstein's special relativity may work only approximately in systems of interacting particles. Here 


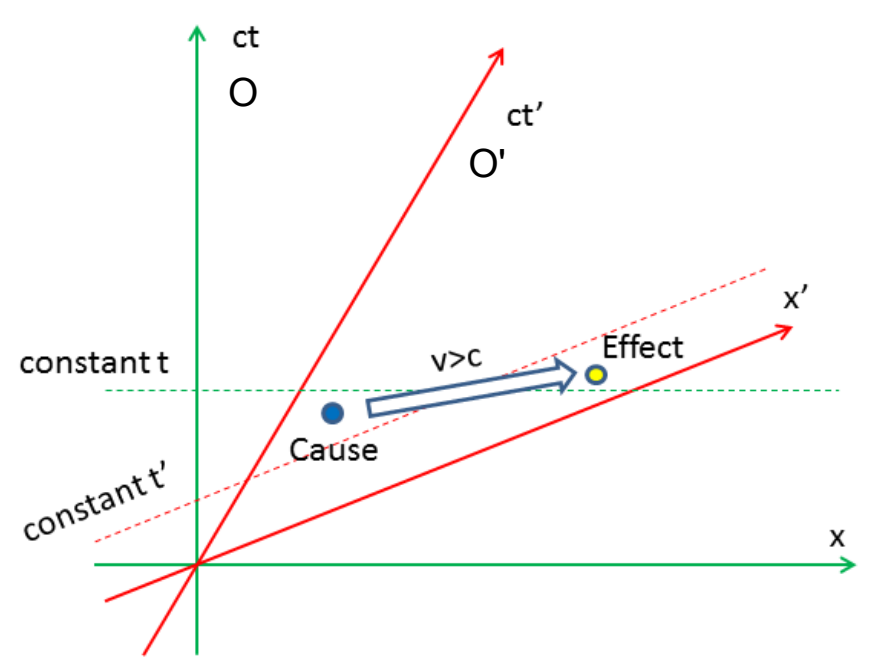

Figure 2: Illustration to the usual "proof" that superluminal signals violate the principle of causality.

we will discuss several specific examples of such systems. In subsection 2 . that Lorentz formulas $(\mathbb{L} \cdot 3)$ - (년) are not exactly applicable to a 2-particle interacting system. In 2.2 we will present a model of oscilating neutrinos that can move faster than the speed of light. In 2.3 we will see that the decay law of fast moving unstable particles may not agree with Einstein's time dilation formula. An experiment that can prove the instantaneous (non-retarded) nature of electromagnetic interactions will be discussed in subsection 2.4.

\subsection{Two-particle system}

Our first example is a two-particle system in one spatial dimension with a fully relativistic interaction potential of the Bakamjian-Thomas type [目]. Equations of motion and boost transformations were solved numerically in the classical limit $\hbar \rightarrow 0$ [].

With appropriate initial conditions, in the rest frame the two particles oscillate around the common center of mass, as shown in Fig. B](a). If we now apply standard Lorentz formulas (ㄴ.3) - ([L.4), we obtain transformed trajectories B(c) that exhibit the usual relativistic effects: a length-contracted oscillation amplitude and a time-dilated oscillation period. However, as we already know, this is not the correct way to switch to the moving reference frame. The true transformation formulas are those involving the interacting boost operator (ㅍ.5). Their application yields moving frame trajectories B(b) that are quite different from the predictions of special relativity. It is remarkable that there are short periods of time when particle's speed exceeds the speed of light. However, in 


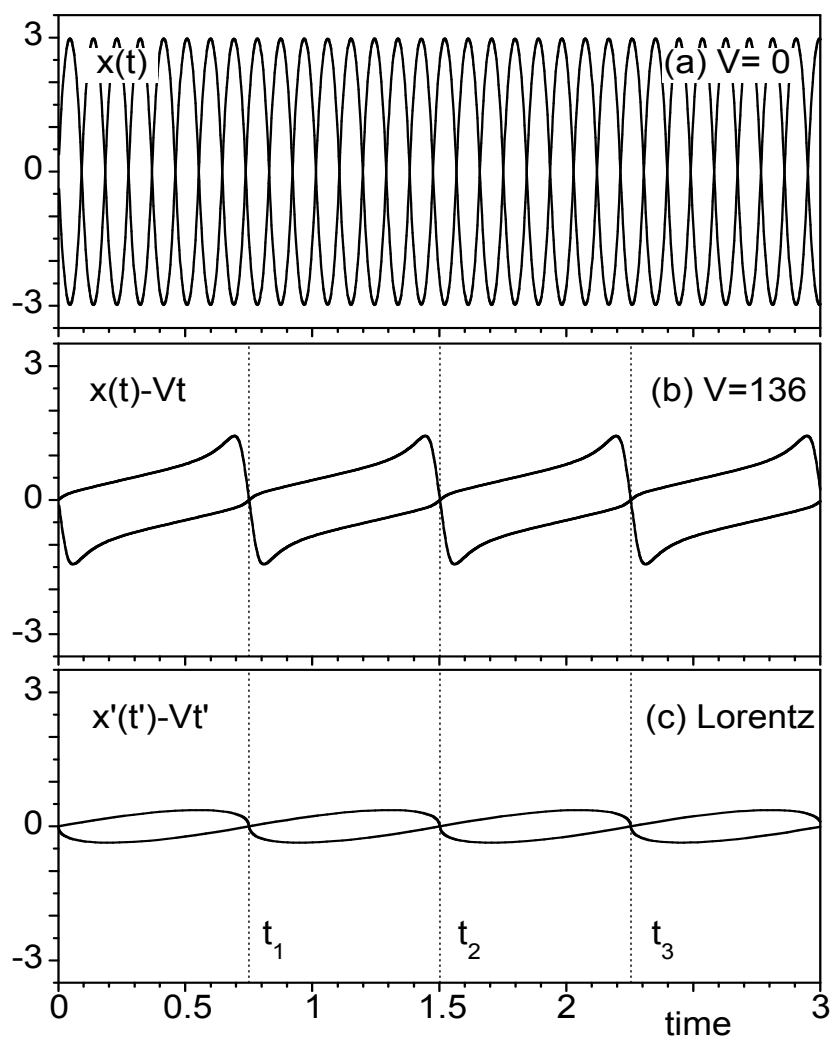

Figure 3: Two particle trajectories $x_{1}(t)$ and $x_{2}(t)$ as functions of time (a) in the rest frame; (b) transformed to the moving frame by formula (1.5); (c) transformed to the moving frame by Lorentz formulas (1.3) - (1.4). In panels (b) and (c) the drift term $V t$ has been subtracted out.

agreement with the principle of causality, there are no reference frames in which the particle moves "backward" in time.

\subsection{Oscillating neutrinos}

A fully relativistic, albeit one-dimensional, model of $v_{\mu}-v_{\tau}$ neutrino oscillations was constructed in $[\mathbb{8}]$. See also reference $[Q]$. The two-level momentum-dependent Hamiltonian in the flavor basis is

$$
H=H_{0}+V=\left[\begin{array}{cc}
\Omega_{\mu}(p) & f(p) \\
f(p) & \Omega_{\tau}(p)
\end{array}\right]
$$

The conditions of Hermiticity and relativistic invariance can be fulfilled if matrix elements $f(p)$ and $\Omega_{\tau}(p)$ are certain functions of $\Omega_{\mu}(p)$, while the latter matrix element satisfies two inequalities

$$
\sqrt{m_{2}^{2} c^{4}+p^{2} c^{2}} \leq \Omega_{\mu}(p) \leq \sqrt{m_{3}^{2} c^{4}+p^{2} c^{2}}
$$

where $m_{2}$ and $m_{3}$ are two neutrino mass eigenvalues. 


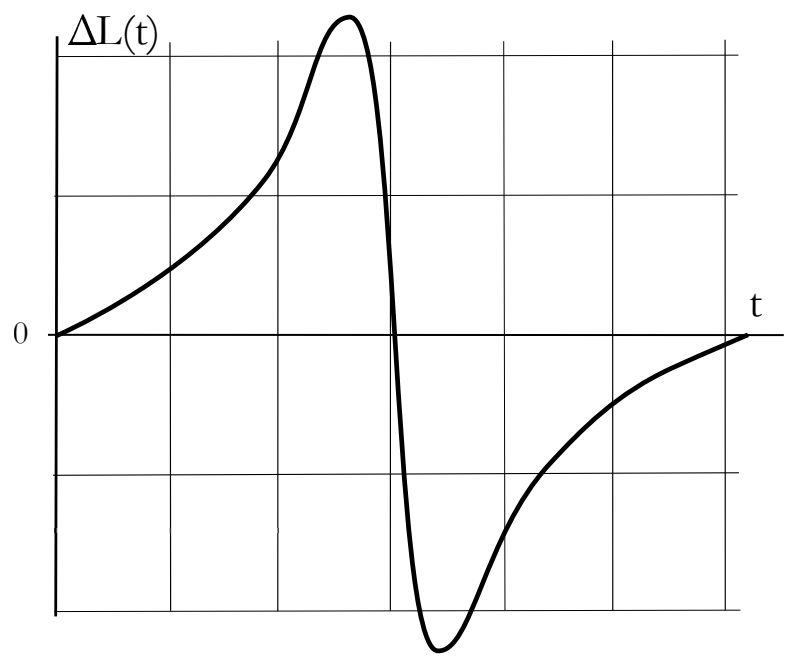

Figure 4: Oscillations of the $v_{\mu}$ neutrino trajectory.

For further analysis it is convenient to adopt the classical limit and calculate trajectories of the two neutrino species as time-dependent expectation values of their position operators. As expected, the center of mass of the neutrino system moves with a constant velocity that is slightly lower than the speed of light $X_{c . m .}(t) \approx c t$. It is more surprising that the $\mu$-neutrino component oscillates around this average path

$$
\begin{aligned}
& x_{\mu}(t) \approx X_{c . m .}(t)+\Delta L(t) \\
& \Delta L(t)=\frac{\hbar}{\gamma \rho_{\mu}(t)}\left(\frac{d \Omega_{\mu}}{d p}-c\right) \sin \frac{\gamma t}{\hbar}
\end{aligned}
$$

where $\gamma(p) \equiv\left(m_{3}^{2}-m_{2}^{2}\right) c^{3} /(2 p)$ and

$$
\rho_{\mu}(t)=1-\sin ^{2}\left(2 \theta_{23}\right) \sin ^{2} \frac{\gamma t}{2 \hbar}
$$

is the probability of finding the $\mu$-neutrino at time $t$. Function $\Delta L(t)$, describing the deviation of the $v_{\mu}$ trajectory from the uniform linear path $X_{c . m .}(t)$, is plotted in Fig. 目. For a half-period $\Delta L(t)>0$, and the $v_{\mu}$ species moves ahead of the center-of-mass trajectory. Then it slows down and lags behind for another half-period. This corresponds to negative values of $\Delta L(t)$. This pattern repeats indefinitely in a periodic fashion. In principle, at certain time intervals the velocity of one neutrino species can exceed the speed of light $c$. However, according to the condition (R.d), the value of $d \Omega_{\mu} / d p$ cannot be very different from the speed of light $c$. So, the magnitude of the factor $\left(d \Omega_{\mu} / d p-c\right)$ in (L.2) is very small, and the superluminal effect is likely to be undetectable.

\subsection{Moving unstable particles}

Another relativistic example in which predictions of the Einstein's theory do not hold, is the

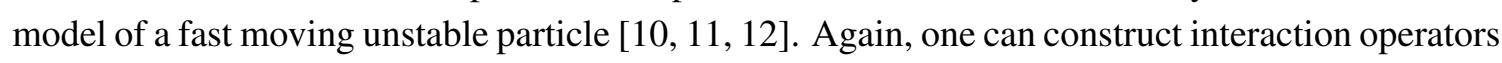




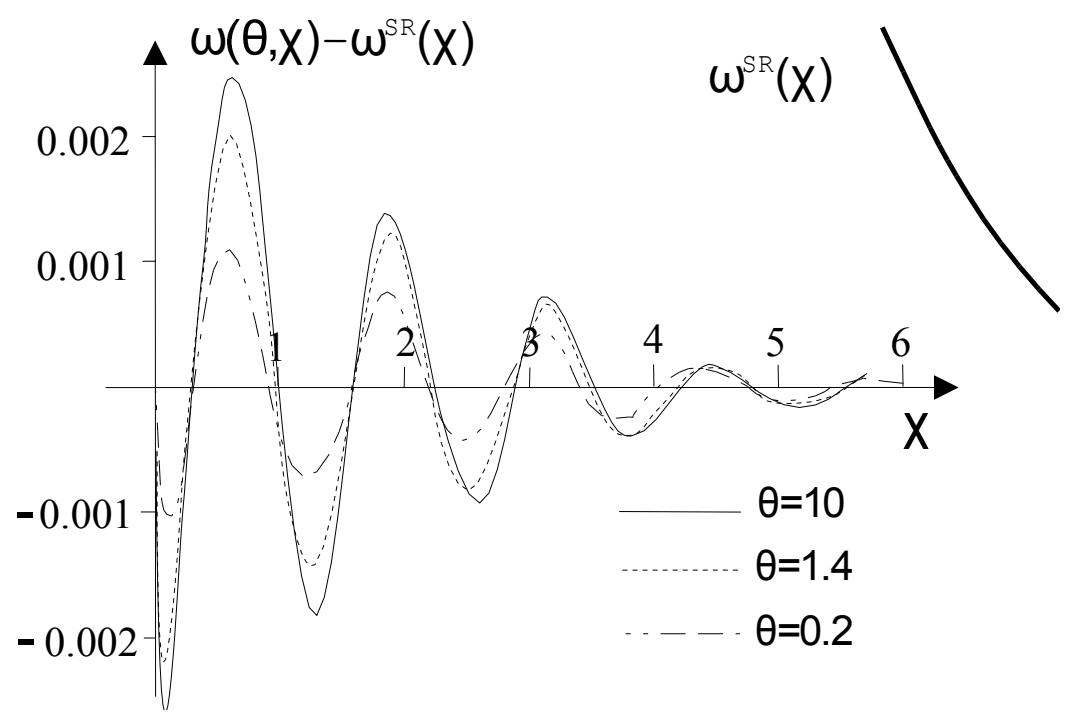

Figure 5: Corrections to the Einstein's "time dilation" formula (2.3) for the decay law of an unstable particle moving with the speed $v=c \tanh \theta$. Parameter $\chi$ is time measured in units of $\cosh \theta / \Gamma$.

$V$ and $Z$, such that Poincare commutation relations remain valid. With the Hamiltonian $H=H_{0}+V$, one gets the decay law at rest, which can be approximated quite well by the usual exponential formula $\omega(t)=\exp (-\Gamma t)$. If we assumed that Einstein's special relativity applies to this case, we would expect that in the moving frame the decay law is given by the same exponent albeit modified by universal "time dilation"

$$
\omega^{S R}(\theta, t)=e^{-\Gamma t / \cosh \theta} \equiv \omega^{S R}(\chi)
$$

where we introduced notation $\chi \equiv \Gamma t / \cosh \theta$. (This decay law $\omega^{S R}(\chi)$ for all rapidities $\theta$ is shown by the thick line in Fig. [1.) However, a more careful transformation, involving the interacting boost operator, results in the true decay law $\omega(\theta, \chi)$ that is different from the Einstein's prediction $([2.3)$. The calculated corrections $\omega(\theta, \chi)-\omega^{S R}(\chi)$ for three values of $\theta$ are show by thin lines in Fig. 1 . In our model system they can exceed $0.2 \%$. However, for more realistic examples, like the unstable $\mu$-meson, the corrections are estimated to be many orders of magnitude smaller, i.e., below the resolution of modern experimental techniques.

\subsection{Experiment at Frascati}

In our model in subsection [ل], the two particles interacted via an instantaneous action-at-adistance potential. According to the usual interpretation of relativity, such interactions are forbidden, and the "true" interaction must be retarded, i.e., propagating with the speed of light. For example, in classical electrodynamics the instantaneous Coulomb potential between charged particles is regarded as a mere approximation to the presumably exact retarded Liénard-Wiechert potential. The special-relativistic ban on instantaneous direct interactions was based on the same combina-

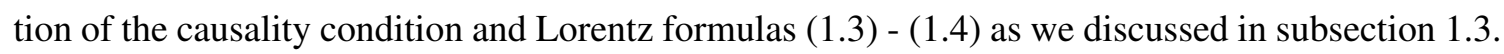

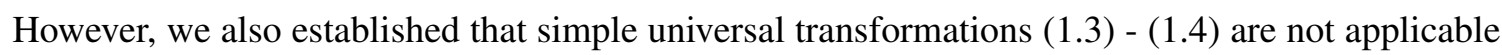



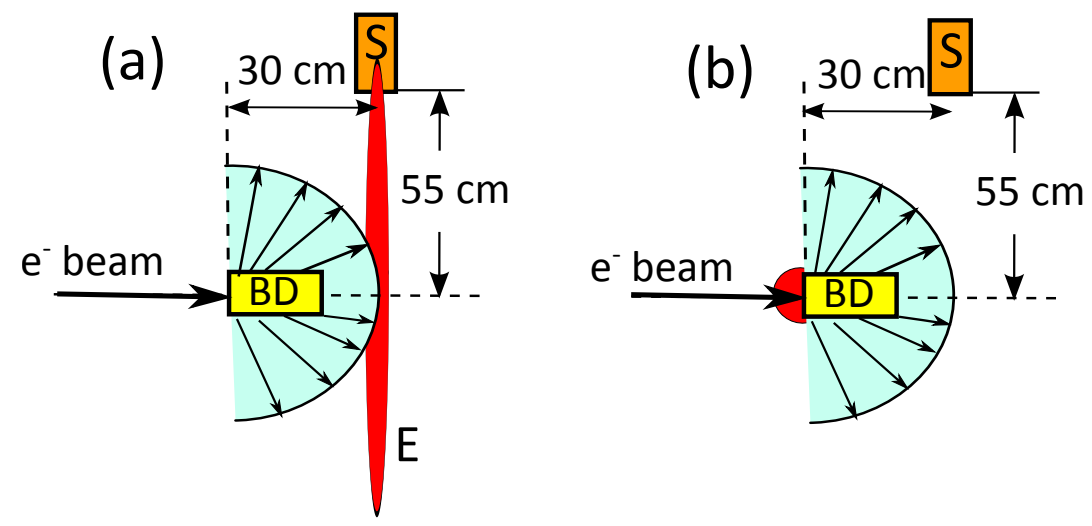

Figure 6: Field configurations at $t=1 \mathrm{~ns}$, i.e., after the electron bunch was stopped by the beam dump $(B D)$ : (a) Maxwell's theory in which the disk-shaped electric field $E$ of the beam has reached the sensor $(S)$; (b) Dressed particle QED in which the runaway disk-shaped electric field is absent (a much weaker stationary field surrounds the stopped electron bunch), and the photons emitted from the collision point have not reached the sensor yet.

in systems of interacting charges. Thus the usual argumentation against action-at-a-distance does not work.

On the other hand, there are convincing theoretical reasons to think that interactions between charged particles depend only on their instantaneous positions and momenta. Such action-at-adistance potentials are normally obtained within the "dressed particle" formulation [ए3], [4]] of quantum electrodynamics. Moreover, the idea of the instantaneous Coulomb potential that is rigidly attached to the moving charge was supported by a remarkable experiment performed by the group of prof. Guido Pizzella at Frascati [155].

We would like to suggest a simple modification of the Frascati experiment, which may provide even more spectacular validation of the instantaneous character of Coulomb forces. The proposed experimental setup is shown in Fig. 6: A freely propagating high-energy electron beam enters the picture from the left. There is a beam dump (BD) on the path of the beam and an electric field sensor $S$ located $30 \mathrm{~cm}$ behind the beam dump and $55 \mathrm{~cm}$ off the beam's axis. The laboratory clock is set so that at time $t=0$ the electron beam hits the beam dump and stops. Undisputtably, the electric field of the free propagating beam before the collision (at times $t<0$ ) is strongly compressed in the direction of motion. The field has the form of a narrow "pancake" perpendicular to the beam's axis and moving together with the electron bunch. The question is what will happen to the electric field at $t>0$, i.e., after the collision, and what will the sensor $S$ measure? Let us now discuss predictions of the two competing theories: the traditional Maxwell's theory with retarded Liénard-Wiechert potentials and the dressed particle QED with instantaneous Coulomb interactions.

In the traditional approach, due to the retarded character of the Liénard-Wiechert field, the information about the beam's halt cannot reach remote parts of the field immediately. So, the electric field "pancake" will continue its motion largely unaffected. This means that the sensor $S$ will register the onset of the field pulse at time $t=30 \mathrm{~cm} / \mathrm{c}=1 \mathrm{~ns}$, as if the beam dump was absent. See Fig. G(a). The bunch's collision with the beam dump will also result in a burst of electromagnetic radiation, which will propagate radially from the collision point with the speed 
of light. The distance between the collision point and the sensor is $\approx 64 \mathrm{~cm}$. Therefore, the electromagnetic pulse will reach the sensor only at time $t>2 \mathrm{~ns}$.

In the dressed particle QED approach [ㅍ], the electric field configuration immediately follows the charges' trajectory. So, after the electron bunch stops at $t=0$, its Coulomb field suddenly transforms into a spherically symmetric shape, characteristic for a charge at rest. See Fig. G(b). At the same time, the field strength drastically weakens below the detector's sensitivity. Thus, we do not predict any response of the sensor $S$ at $t=1 \mathrm{~ns}$. Formation of the bremsstrahlung photon pulse at $t=0$ will proceed as described above, and this signal will reach the sensor only after $t>2 n s$.

As we see, predictions of the two theories are quite different. They disagree about the timing of the signal onset in $S$ by as much as $1 n s$. This difference can be easily detected within the Frascati experimental setup.

\section{Conclusions}

1. In interacting systems one needs to take into account corrections to usual formulas of Einstein's special relativity.

2. The best chance for an experimental confirmation of these corrections is provided by the Frascati experiment with energetic electron beams.

\section{References}

[1] E. P. Wigner. On unitary representations of the inhomogeneous Lorentz group. Ann. Math. 40 (1939) 149.

[2] T. D. Newton and E. P. Wigner. Localized states for elementary systems. Rev. Mod. Phys. 21 (1949) 400.

[3] E. V. Stefanovich. Is Minkowski space-time compatible with quantum mechanics? Found. Phys. 32 (2002) 673.

[4] P. A. M. Dirac. Forms of relativistic dynamics. Rev. Mod. Phys. 21 (1949) 392.

[5] D. G. Currie, T. F. Jordan and E. C. G. Sudarshan. Relativistic invariance and Hamiltonian theories of interacting particles. Rev. Mod. Phys. 35 (1963) 350.

[6] B. Bakamjian and L. H. Thomas. Relativistic particle dynamics. II. Phys. Rev. 92 (1953) 1300.

[7] B. T. Shields, M. C. Morris, M. R. Ware, Q. Su, E. V. Stefanovich and R. Grobe. Time dilation in relativistic two-particle interactions. Phys. Rev. A 82 (2010) 052116.

[8] E. V. Stefanovich. Model of superluminal oscillating neutrinos (2012) http://www.vixra.org/pdf/1201.0091v2.pdf.

[9] B. Sazdović and M. Vasilić. Oscillations of neutrino velocity. JHEP 03 (2013) 47 [arXiv:1210.3531].

[10] E. V. Stefanovich. Quantum effects in relativistic decays. Int. J. Theor. Phys. 35 (1996) 2539.

[11] M. I. Shirokov. Evolution in time of moving unstable systems. Concepts of Physics 3 (2006) 193 [quant-ph/0508087].

[12] K. Urbanowski. Decay law of relativistic particles: Quantum theory meets special relativity. Phys. Lett. B 737 (2014) 346 [arXiv:1408.6564]. 
[13] A. V. Shebeko and M. I. Shirokov. Unitary transformations in quantum field theory and bound states. Phys. Part. Nucl. 32 (2001) 15 [nucl-th/0102037].

[14] E. V. Stefanovich. Relativistic quantum dynamics. physics/0504062.

[15] A. Calcaterra, R. de Sangro, G. Finnochiaro, P. Patteri, M. Piccolo and G. Pizzella. Measuring propagation speed of Coulomb fields. arXiv:1211.2913. 